\title{
The influence of age, sex and visual dysfunction on reading ability in school-children
}

\author{
António M. G. Baptista ${ }^{1,}{ }^{*}$, Pedro Serra ${ }^{2}$, Carlos Silva ${ }^{3}$, Sandra Franco ${ }^{1}$, James Gilchrist ${ }^{4}$, Brendan Barrett ${ }^{5}$ \\ ${ }^{1}$ University of Minho, Centre of Physics, Rua da Universidade 4710 - 057 Braga, Portugal \\ ${ }^{2}$ Instituto Superior de Educação e Ciência, Alameda das Linhas de Torres 179, 1750-142 Lisboa, Portugal \\ ${ }^{3}$ Clinical Practice, Braga, Portugal \\ ${ }^{4}$ Senior Researcher, UK \\ ${ }^{5}$ University of Bradford, Optometry Department, Richmond Rd, Bradford BD7 1DP, UK
}

\begin{abstract}
Binocular and accommodative dysfunctions affect the efficiency and coordination of the eyes, and this may impair reading performance. Reading can be evaluated using accuracy and speed measures. In this study, we searched for evidence that dysfunctions of accommodation (i.e. accommodation insufficiency, IA) and binocular vision (i.e. convergence insufficiency, $\mathrm{CI}$ ) and also hyperopia have impact on reading ability. A sample of 292 children read a text following vision screening. The sample was composed of control children and children with the conditions: AI; CI and hyperopia. Multilinear regression analysis was used to evaluate the influence of variables sex, age and visual condition (control, AI, CI and hyperopia) on reading accuracy and speed. The results showed that $\sim 7 \%$ of the variation in reading accuracy was explained by a model where age $(\mathrm{p}=0.001)$, and presence of AI $(\mathrm{p}<0.001)$ and hyperopia $(\mathrm{p}=0.019)$ were statistically significant. On the other hand, $\sim 38 \%$ of the variation in the index of speed was explained by age alone $(p<0.001)$. These results confirm the importance of age on children's ability to read, and suggests that AI and hyperopia, but not CI may affect reading accuracy. Reading speed does not appear to be affected by the presence of binocular or accommodative dysfunction.
\end{abstract}

\section{Introduction}

Binocular and accommodative dysfunctions affect the efficiency and coordination of the eyes, and this may impair reading performance, a critical skill for learning. Reading performance can be evaluated using accuracy and speed measures. Accuracy (1) reflects the percentage of words correctly read [1], and speed (2) corresponds to the number of words correctly read per minute [1]. In this study, we searched for evidence that the most prevalent dysfunctions of accommodation (i.e. accommodation insufficiency, AI) and binocular vision (i.e. convergence insufficiency, CI) and also hyperopia have impact on reading ability.

Accuracy $=\frac{\text { Total number of words read-Total number of errors }}{\text { Total number of words read }}$

Speed $=\frac{\text { Total number of words read-Total number of errors }}{\text { Reading time }} \times 60$

\section{Methods}

Accommodation insufficiency was defined as monocular accommodative amplitude less than 12.5 spherical dioptres. Convergence insufficiency was defined as [near point of convergence equal or greater than $6 \mathrm{~cm}\{\mathrm{AND}\}$ near exophoria at least four prism dioptres greater than distance exophoria $\{A N D\}$ (failing Sheard's criterion $\{\mathrm{OR}\}$ positive fusional vergence equal or less than 15 prism dioptres base-out)]. Hyperopia was defined as being positive on the blur test $(+2.00$ spherical dioptres $)$ in either eyes. A sample of 292 children read a text of a traditional, Portuguese tale "O rei vai nu" [1] following vision screening tested for: autorefractor; monocular visual acuity with habitual refraction; cover test; near point of convergence; blur test +2.00 spherical dioptres; distance phoria; stereopsis (TNO); monocular near visual acuity; monocular accommodative amplitude. All testing took place in the school (29 schools were visited). The sample was composed of control children (absence of AI, CI and hyperopia as defined above, stereopsis equal or better than 120 arc sec and monocular visual acuity equal or better than 0.8 decimal) and children with the conditions: AI; $\mathrm{CI}$ and hyperopia. Multilinear regression analysis was used to evaluate the influence of variables sex (binary variable: male $=0$ and female $=1$ ), age and visual condition (nominal variable: control, AI, CI and hyperopia; e.g. when $\mathrm{AI}=1$ then control $=0$ and $\mathrm{CI}=0$ and hyperopia $=0$ ) on reading accuracy and speed.

This research was conducted according to the guidelines outlined in the Declaration of Helsinki. Written informed consent was obtained from children parents. This study

\footnotetext{
* Corresponding author: abaptista@fisica.uminho.pt
} 
received ethical approval by the Ethics Subcommittee for Life and Health Sciences of Minho University.

\section{Results}

Number of control children: male: $n=91,9.7 \pm 1.4$ years; female: $n=85,9.6 \pm 1.3$ years. Number of children with the conditions AI: male: $n=48,10.1 \pm 1.4$ years; female: $n=36$, $10.8 \pm 1.6$ years. Condition CI: male: $n=5,9.5 \pm 1.8$ years; female: $n=6: 10.0 \pm 2.0$ years. And hyperopia: male: $n=5$, $8.9 \pm 2.2$ years; female: $n=15,9.5 \pm 1.5$ years. The results showed that $\sim 7 \%$ of the variation in reading accuracy was explained by a model where age $(\mathrm{p}=0.001)$, and presence of AI $(p<0.001)$ and hyperopia $(p=0.019)$ were statistically significant but not $\operatorname{sex}(\mathrm{p}=0.954)$ or CI $(\mathrm{p}=0.344)$. On the other hand, $\sim 38 \%$ of the variation in the index of speed was explained by age alone $(p<0.001)$, i.e. the presence or absence of hyperopia $(p=0.598)$, AI $(p=0.294)$ or $\mathrm{CI}(\mathrm{p}=0.565)$ or $\mathrm{sex}(\mathrm{p}=0.384)$ did not affect speed.

\section{Conclusions}

The results confirm the importance of age on children's ability to read, and suggests that accommodation insufficiency and hyperopia, but not convergence insufficiency may affect reading accuracy. Reading speed does not appear to be affected by the presence of binocular or accommodative dysfunction.

\section{Acknowledges}

This work was supported by the Portuguese Foundation for Science and Technology (FCT) in the framework of the Strategic Funding UID/FIS/04650/2013.

\section{References}

1. Anabela O.D.C. Carvalho, Teste de avaliação da fluência e precisão de Leitura : O Rei (EDIPSICO, 2010) 